\title{
An Analysis of Scheimpflug Holladay-Equivalent Keratometry Readings Following Corneal Collagen Cross-Linking
}

\author{
(D) Ayhan Saglik, ${ }^{1}$ (1) Hakim Celik, ${ }^{2}$ (1) Mustafa Aksoy ${ }^{1}$ \\ 1'Department of Ophthalmology, Harran University, Sanliurfa, Turkey \\ ${ }^{2}$ Department of Physiology, Harran University, Sanliurfa, Turkey
}

\begin{abstract}
Objectives: The aim of this research was to compare Holladay equivalent keratometry readings (EKR) and anterior corneal simulated keratometry (SimK) values in keratoconus cases following corneal collagen cross-linking (CXL) treatment.

Methods: This study included 42 eyes of 30 patients with progressive keratoconus. Scheimpflug imaging was performed pre-CXL and at post-CXL months 6 and I2. The mean Holladay EKR and SimK measurements from Imm to $7 \mathrm{~mm}$ were compared and repeated variance analysis was performed.

Results: The mean age of the patients was 18.93 \pm 3.82 years (range: 12-27 years). A reduction was observed in the EKR and SimK values at 6 and 12 months postoperatively compared with the preoperative values, but the difference was not statistically significant $(p>0.05)$. In the mild keratoconus group, the keratometric measurements were lower after treatment. Similar measurements were obtained in the moderate and severe keratoconus groups before and after treatment. In I patient, there was an increase of $>$ I diopter in the maximum keratometry value. No complications developed that would lead to vision loss.

Conclusion: Corneal collagen CXL treatment is known to be effective to a depth of two-thirds of the anterior corneal stroma. The results of this study showed that Holladay EKR measurements differed from SimK measurements, especially in cases of mild keratoconus. In addition to anterior corneal surface measurements, methods that can evaluate anterior and posterior surface curves could provide more accurate results in progression analysis.
\end{abstract}

Keywords: Cornea, crosslinking, holladay equivalent keratometry readings, scheimpflug; simulated keratometry.

\section{Introduction}

Keratoconus is a progressive ectatic disease of the cornea that can lead to irregular astigmatism and vision loss (I). Advanced stage keratoconus can result in a need for keratoplasty. Topographic parameters of the cornea are currently widely used to identify early-stage keratoconus, and are also important in the follow-up of the progression of keratoconus (2).

Corneal collagen cross-linking $(\mathrm{CXL})$ treatment has been demonstrated to be effective and reliable. Several studies in the literature have examined the efficacy of CXL (3-7). CXL treatment has resulted in stabilization or regression in keratometric, tomographic, and refractive findings, and there may be an increase in visual acuity. The aim of strengthening the anterior corneal stroma with CXL treatment is to increase corneal rigidity and halt the progression of keratoconus $(8,9)$. Topographic examinations are widely used in the follow-up of clinical progression.

Address for correspondence: Ayhan Saglik, MD. Osmanbey Kampusu, Harran Universitesi Tip Fakultesi, Hastanesi Goz Hastaliklari Poliklinigi Haliliye, Sanliurfa, Turkey Phone: +90 5363561171 E-mail: saglikayhan@yahoo.com 
Equivalent keratometry readings (EKR), defined by Holladay et al., (I0) were developed as measurements to calculate the power of the intraocular lens $(\mathrm{IOL})$ in patients undergoing corneal refractive surgery. In addition to local corneal thickness measurements, the Pentacam HR system (Oculus $\mathrm{GmbH}$, Wetzlar, Germany), which includes a Scheimpflug camera system, records Holladay EKR measurements using anterior and posterior corneal surface elevation values (10-12).

The aim of this study was to compare Holladay EKR and anterior corneal simulated keratometry (SimK) values following corneal collagen cross-linking $(\mathrm{CXL})$ treatment in keratoconus cases calculated using corneal anterior and posterior surface power measurements documented in the 12-month follow-up period.

\section{Methods}

This study included 42 eyes of 30 patients who were diagnosed with progressive keratoconus, treated with corneal collagen CXL, and followed up in the Cornea Unit of the Ophthalmology Department of Harran University Medical Faculty Hospital. Approval for the study was granted by the Ethics Committee of the Faculty of Medicine of Harran University, Şanliurfa, Turkey. All of the study procedures were performed in compliance with the Helsinki Declaration.

The diagnosis of keratoconus was made based on findings of Fleischer's ring, Vogt's striae, and stromal thinning, using classic slit-lamp biomicroscopy, Pentacam Scheimpflug images and topography findings, such as an asymmetric bow-tie pattern and inferior-superior asymmetry. Keratoconus progression was confirmed based on the evaluation of corneal topography, visual acuity, and pachymeter and manifest refraction measurements. Progression was defined as an increase of $>$ I diopter (D) in the maximum keratometry $(K \max )$ value within 12 months.

Patients were excluded from the study if the thinnest corneal thickness was $<400 \mu \mathrm{m}$ on Scheimpflug images, if there was excessive axial corneal scarring, ocular trauma, a history of ocular surgery, herpetic keratitis, autoimmune disease, or contact lens use within the last month. Keratoconus grading was performed using the Amsler-Krumeich classification. The patients were evaluated in 3 groups: mild $(K<48 D)$, moderate (K 48-53D), and severe $(K>53 \mathrm{D})$ according to the mean keratometry $(K)$ value obtained from Scheimpflug topography images. (12, I3).

\section{Scheimpflug Evaluation}

Corneal topography evaluation was performed for all patients before treatment and at 6 and 12 months postoperatively (Pentacam HR, software version: I.2Ir3). The most reliable measurements were recorded, as well as the mean SimK and Holladay EKR Detail Report measurements.
SimK is an estimate of total corneal strength as a single refracting surface based on the anterior cornea curvature and the standard corneal refraction index of the cornea. The values were calculated using the formula $\operatorname{SimK}=n-n 0 /$ ranterior when ranterior is the anterior central corneal curvature, within a certain diameter (in meter), $\mathrm{n} 0$ is the refractive index of air (1.000), and $\mathrm{n}$ is the standard corneal refractive index (I.3375) $(14,15)$.

The Holladay EKR software was developed to calculate the total corneal power of patients undergoing corneal refractive surgery (10). The Holladay EKR Detail Report obtained using the Pentacam HR device takes measurements at $1 \mathrm{~mm}$ intervals of the central corneal diameter, starting at I $\mathrm{mm}$ and continuing to $7 \mathrm{~mm}$. A $4.5 \mathrm{~mm}$ central zone is measured using the clinical history method. The EKR can be calculated using the Pentacam standard refractive index and the radii of the anterior and posterior corneal surfaces. The values were calculated using the formula of EKR $(D)=0.376 /$ ranterior $-0.03165 /$ rposterior when rposterior is the posterior central corneal curvature, within a certain diameter (in meter).

\section{Surgical Technique}

The standard CXL procedure, as described by Wollensak et al. (8), was performed in all of the study cases. Anesthesia was provided with $0.5 \%$ topical proparacaine hydrochloride and followed by $9.0 \mathrm{~mm}$ of mechanical debridement of the corneal epithelium. After ultrasonic pachymeter measurement, isotonic riboflavin (0.1\% riboflavin with $20 \%$ dextran T500; Collagex, Taipei, Taiwan) was administered with drops at 2-minute intervals for $30 \mathrm{~min}$.

Riboflavin absorption in the anterior chamber was checked with a slit-lamp biomicroscope. The central corneal thickness measurement was recorded with an ultrasound pachymeter (UP), and hypotonic riboflavin $(0.1 \%$ in sterile water; Collagex, Taipei, Taiwan) was administered with I drop every 10 seconds for 2 minutes when the thickness was $<400 \mu \mathrm{m}$. UP was used again to confirm a corneal stromal thickness. After obtaining an appropriate sample, UVA light of $365 \mathrm{~nm}$ (LightLink-CXL, LightMed Corp., San Clemente, CA, USA) was applied at a radiance of $3.0 \mathrm{~mW} / \mathrm{cm}^{2}$ for 30 minutes. When hypotonic riboflavin was required, I drop every 2 min was administered during the UVA application.

A soft contact lens was applied postoperatively and topical antibiotic drops were used 4 times per day for I week. After epithelial healing, the contact lens was removed and topical steroid treatment was initiated at 4 times per day for 2 weeks and continued for up to 3 months with a tapering dosage.

\section{Statistical Analysis}

Data obtained in the present study were statistically analyzed using IBM SPSS Statistics for Windows, Version 22.0 
(IBM Corp., Armonk, NY, USA). Conformity of the data to normal distribution was tested with the Shapiro-Wilk test. Data with normal distribution were described using mean $\pm S D$, and median and interquartile range values were used for data without normal distribution. Repeated variance analysis was applied to determine the effect of time on parameters with normal distribution and the Friedman test was applied to parameters not showing normal distribution. Heat map analysis was performed to examine the distribution of all of the data. A value of $p<0.05$ was accepted as statistically significant.

\section{Results}

This study included 42 eyes ( 2 I right, 21 left) of 30 patients with progressive keratoconus. The group comprised I5 (50\%) males and I5 (50\%) females, with a mean age of $18.93 \pm 3.82$ years (range: 12-27 years). The keratoconus was classified as severe in 10 (24\%) eyes, moderate in $18(43 \%)$, and mild in $14(33 \%)$. Localization of the conus was central in 28 (66\%) eyes and paracentral in 14 (34\%) eyes. Allergic conjunctivitis in 4 patients was treated with medication fol- lowed by surgery. Hypotonic riboflavin solution was used in $4(9.5 \%)$ eyes.

The mean Kmax value was measured at 56.4ID preoperatively and $56.06 \mathrm{D}$ at the postoperative 12 -month follow-up examination $(p=0.083)$.

The preoperative and postoperative Holladay EKR values of the different keratoconus groups are presented in Figure I. No statistically significant difference was observed in the keratometric values at 6 and 12 months postoperatively compared with the preoperative values $(p>0.05)$ (Table I, Table 2). The keratometric measurements of the mild keratoconus group decreased after treatment. Measurements similar to the preoperative values were obtained in the moderate and severe groups. The differences that developed at postoperative 6 and 12 months in the mean EKR and SimK values at different diameters are illustrated in Figure I and Figure 2. In addition, statistical analysis of the difference between Sim K and EKR values was performed. The SimK corneal curvature at all measurement intervals was significantly greater than that of EKR $(p<0.00 I)$.

Holladay EKR, SimK, and Kmax values are shown in a heat

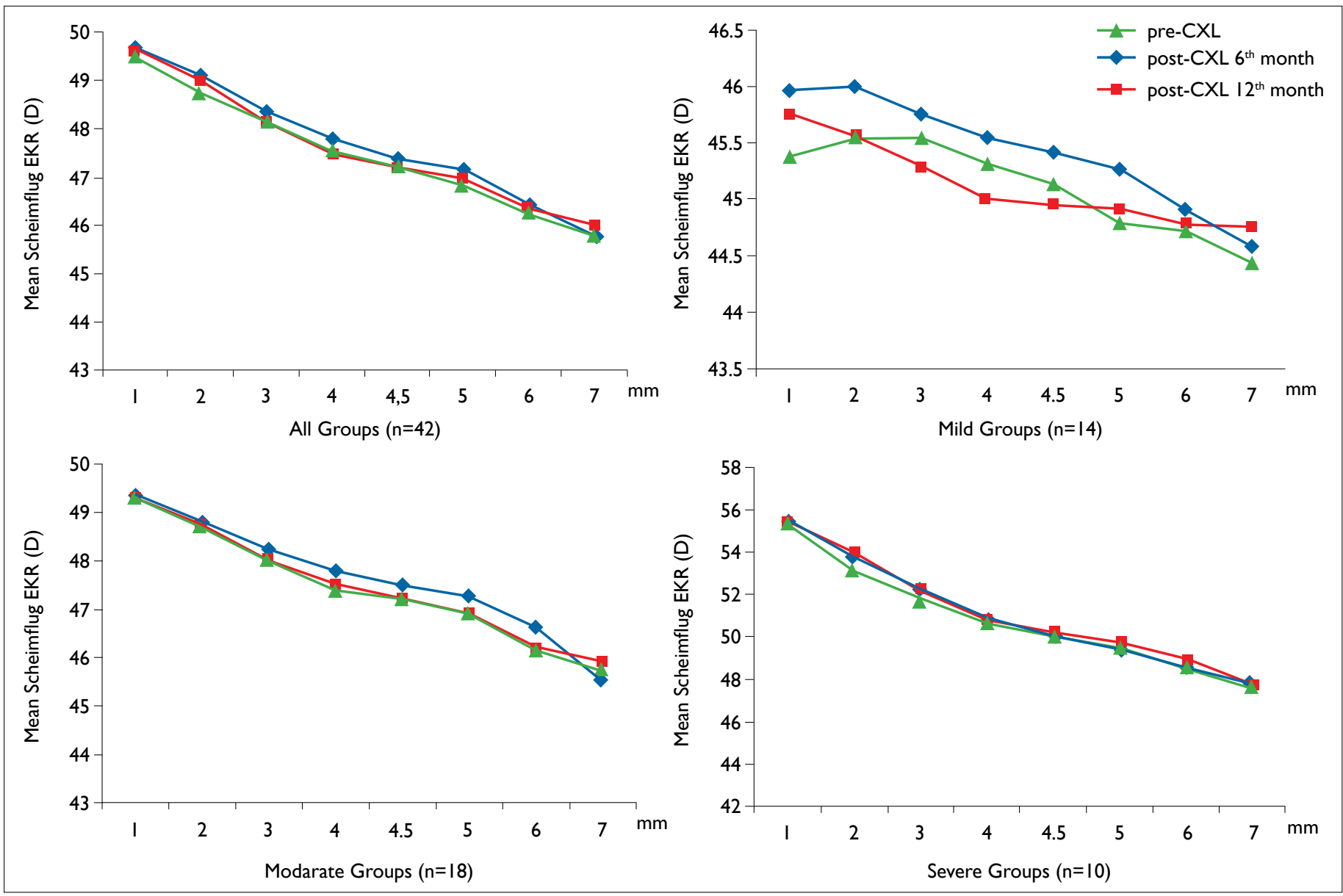

Figure I. Scheimpflug equivalent keratometry reading value in different keratoconus groups before and after corneal cross-linking treatment. CXL: corneal cross-linking; EKR: equivalent keratometry reading. 
Table I. Mean Scheimpflug Holladay EKR measurements before and after CXL treatment

\begin{tabular}{|c|c|c|c|c|c|c|c|c|}
\hline Mean (D) $\pm S D$ & EKR I mm & EKR 2 mm & EKR $3 \mathrm{~mm}$ & EKR $4 \mathrm{~mm}$ & EKR $4.5 \mathrm{~mm}$ & EKR $5 \mathrm{~mm}$ & EKR $6 \mathrm{~mm}$ & EKR $7 \mathrm{~mm}$ \\
\hline Pre-CXL & $49.68 \pm 0.71$ & $49.09 \pm 0.58$ & $48.38 \pm 0.52$ & $47.79 \pm 0.50$ & $47.40 \pm 0.49$ & $47.14 \pm 0.49$ & $46.43 \pm 0.45$ & $45.75 \pm 0.40$ \\
\hline $6^{\text {th }}$ month & $49.63 \pm 0.72$ & $48.97 \pm 0.63$ & $48.12 \pm 0.56$ & $47.45 \pm 0.53$ & $47.19 \pm 0.51$ & $46.94 \pm 0.48$ & $46.39 \pm 0.45$ & $45.98 \pm 0.39$ \\
\hline $12^{\text {th }}$ month & $49.49 \pm 0.70$ & $48.7 I \pm 0.55$ & $48.13 \pm 0.49$ & $47.49 \pm 0.47$ & $47.18 \pm 0.46$ & $46.82 \pm 0.45$ & $46.24 \pm 0.4 I$ & $45.75 \pm 0.39$ \\
\hline$\Delta$ Pre-6 $6^{\text {th }}$ & $0.05 \pm 0.28$ & $0.12 \pm 0.24$ & $0.26 \pm 0.25$ & $0.33 \pm 0.25$ & $0.21 \pm 0.23$ & $0.19 \pm 0.22$ & $0.03 \pm 0.23$ & $-0.22 \pm 0.22$ \\
\hline$\Delta$ Pre- $12^{\text {th }}$ & $0.18 \pm 0.26$ & $0.37 \pm 0.19$ & $0.25 \pm 0.19$ & $0.29 \pm 0.19$ & $0.22 \pm 0.20$ & $0.31 \pm 0.20$ & $0.19 \pm 0.17$ & $00 \pm 0.17$ \\
\hline $\mathrm{P}$ & $=0.882$ & $=0.168$ & $=0.373$ & $=0.228$ & $=0.75 \mathrm{I}$ & $=0.959$ & $=0.636$ & $=0.982$ \\
\hline
\end{tabular}

$\Delta$ Pre $-6^{\text {th }}$ : mean EKR difference between preoperative value and postoperative $6^{\text {th }}$ month; $\Delta$ Pre- $12^{\text {th }}:$ mean EKR difference between preoperative and postoperative $12^{\text {th }}$ month; CXL: corneal cross-linking; EKR: equivalent keratometry reading.

Table 2. Mean Scheimpflug SimK measurements before and after CXL treatment

Mean (D) \pm SD SimK I mm SimK 2 mm SimK 3 mm SimK 4 mm SimK 4.5 mm SimK 5 mm SimK 6 mm SimK 7 mm

\begin{tabular}{lcccccccc}
\hline \hline Pre-CXL & $5 I .20 \pm 0.73$ & $50.79 \pm 0.68$ & $50.16 \pm 0.6 \mathrm{I}$ & $49.40 \pm 0.53$ & $49.00 \pm 0.50$ & $48.58 \pm 0.46$ & $47.53 \pm 0.39$ & $46.96 \pm 0.35$ \\
$6^{\text {th }}$ month & $51.30 \pm 0.73$ & $50.88 \pm 0.68$ & $50.24 \pm 0.62$ & $49.46 \pm 0.55$ & $49.02 \pm 0.5 \mathrm{I}$ & $48.62 \pm 0.48$ & $47.78 \pm 0.4 \mathrm{I}$ & $46.97 \pm 0.36$ \\
$\mathrm{I}^{\text {th }}$ month & $5 \mathrm{I} .17 \pm 0.7 \mathrm{I}$ & $50.75 \pm 0.67$ & $50.1 \mathrm{I} \pm 0.60$ & $49.34 \pm 0.54$ & $48.92 \pm 0.50$ & $48.52 \pm 0.47$ & $47.69 \pm 0.4 \mathrm{I}$ & $46.89 \pm 0.35$ \\
$\Delta$ Pre-6 & $-0.10 \pm 0.18$ & $-0.09 \pm 0.17$ & $-0.08 \pm 0.15$ & $-0.06 \pm 0.13$ & $-0.02 \pm 0.13$ & $0.19 \pm 0.22$ & $-0.25 \pm 0.25$ & $00 \pm 0.09$ \\
$\Delta$ Pre-I2 & $0.03 \pm 0.17$ & $0.04 \pm 0.16$ & $0.04 \pm 0.15$ & $0.06 \pm 0.14$ & $0.07 \pm 0.13$ & $-1.38 \pm 0.36$ & $-0.16 \pm 0.25$ & $0.06 \pm 0.10$ \\
$\mathrm{P}$ & $=0.680$ & $=0.647$ & $=0.604$ & $=0.58 \mathrm{I}$ & $=0.698$ & $=0.650$ & $=0.466$ & $=0.627$
\end{tabular}

$\Delta$ Pre- $6^{\text {th }}$ : mean SimK difference between preoperative value and postoperative $6^{\text {th }}$ month; $\Delta$ Pre $-12^{\text {th }}$ : mean SimK difference between preoperative and postoperative $12^{\text {th }}$ month; CXL: corneal cross-linking; SimK, anterior simulated keratometry measurement.

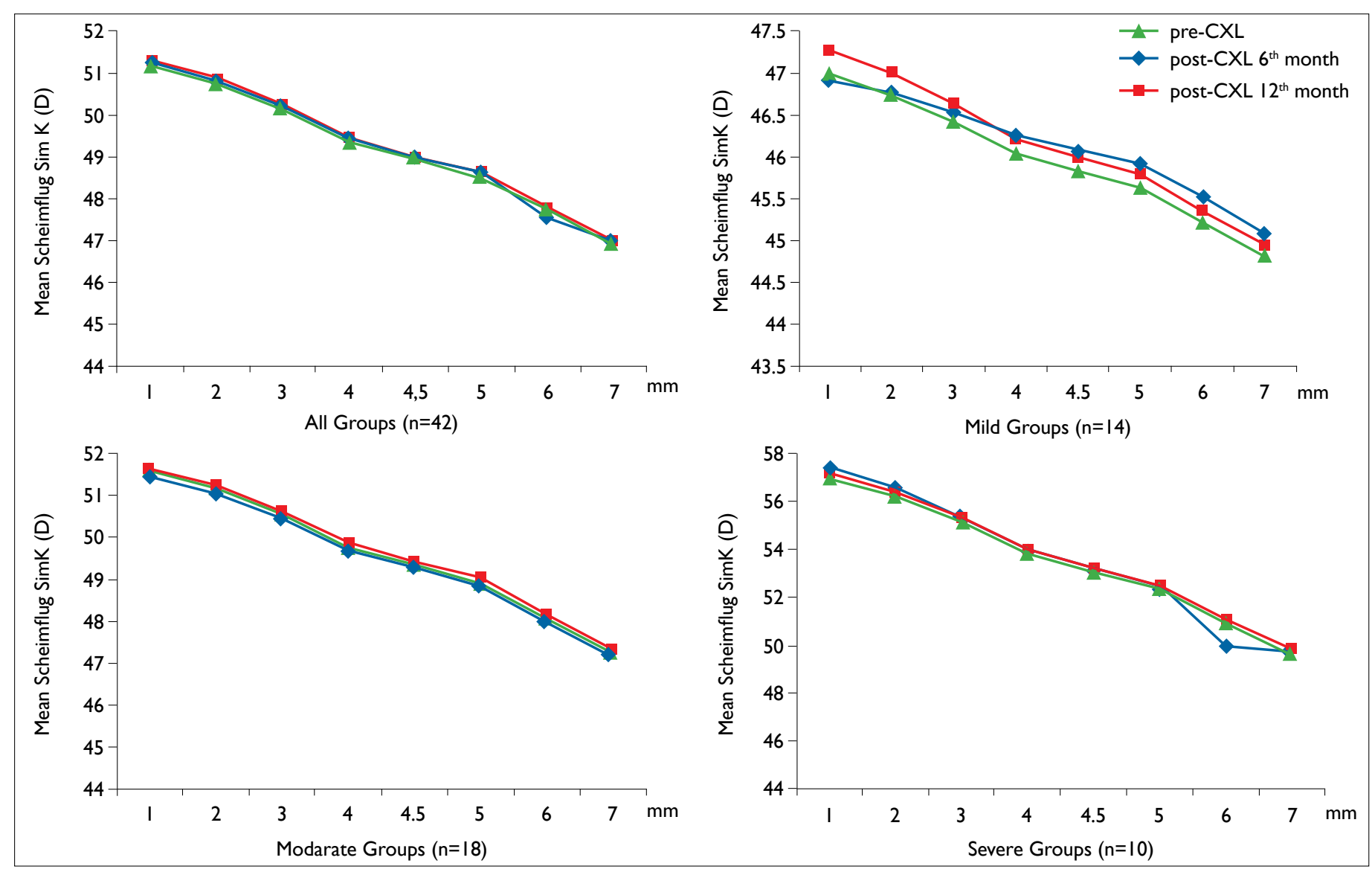

Figure 2. Scheimpflug SimK value in different keratoconus groups before and after corneal cross-linking treatment. CXL: corneal cross-linking; SimK: anterior simulated keratometry measurement. 
map in Figure 3. According to the map, the mean data were distributed homogeneously in the mild and severe groups, whereas in the moderate group, it was more heterogeneous.

Complications such as corneal infiltration or scarring did

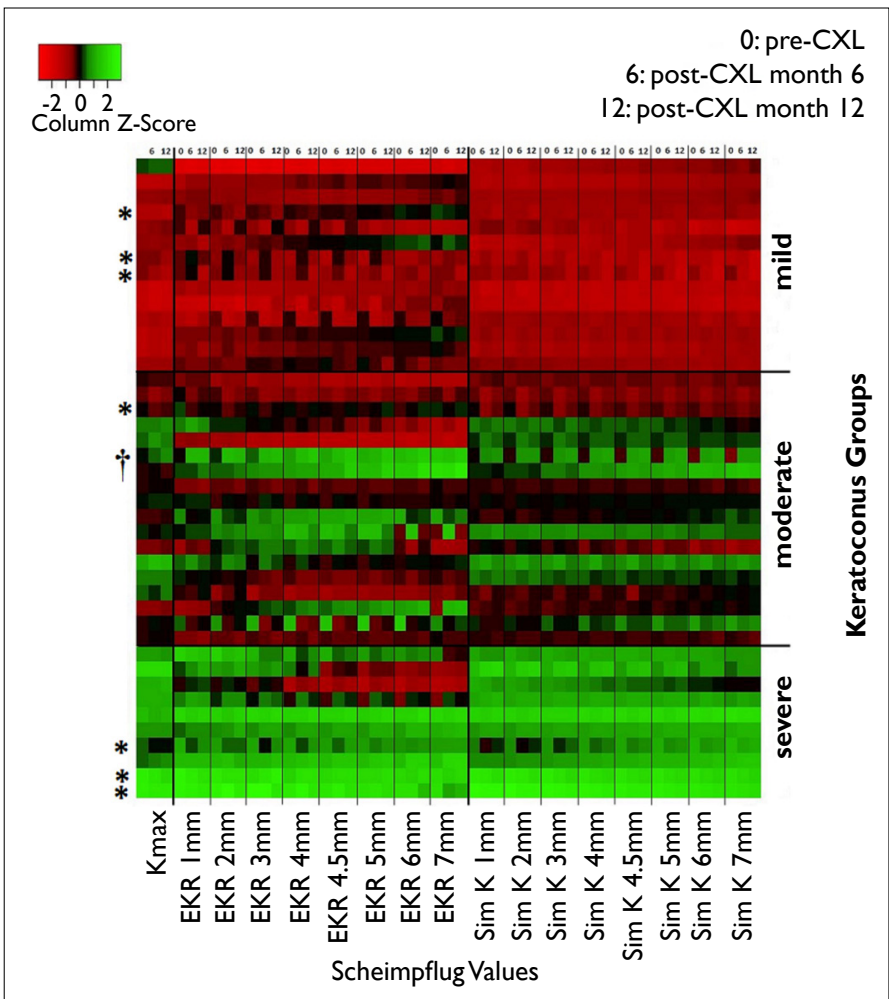

Figure 3. Heat map analysis in mild, moderate, and severe keratoconus groups. EKR: equivalent keratometry reading; Kmax: maximum keratometry; SimK, simulated keratometry. *A corneal flattening of ID in the $\mathrm{Kmax} \dagger$ a corneal steepening of ID in the Kmax. not develop in any eye. At 12 months postoperatively, a decrease of >ID in the Kmax value was determined in 7 eyes: 3 eyes were diagnosed as mild keratoconus, there was I case of moderate keratoconus, and 3 eyes were determined to have severe keratoconus (Fig. 3).

Corneal steepening of >ID in the Kmax was determined in I eye (Fig. 3). The topometric results of this patient can be seen in Figure 4. Increased corneal steepening was seen I month postoperatively but was stable in the sixth month. The best corrected distance visual acuity did not change at 6 and 12 months. Grade I haze was observed in a slit-lamp biomicroscopy examination.

\section{Discussion}

Corneal collagen CXL treatment is currently a method widely used to halt the progression of keratoconus. The efficacy of this treatment has been demonstrated in several studies and different parametric values related to progression have been examined (16-22). In the follow-up period after treatment, topographic parameters can provide guidance. Holladay EKR analysis performed with a device such as the Pentacam HR provides important data used in Scheimpflug topography. EKR measurements were first described by Holladay et al. (I0) and were specifically developed to calculate IOL power after corneal refractive surgery using anterior and posterior surface measurements.

In the current study, a comparison was made of the Holladay EKR measurements and anterior surface SimK values in different corneal ranges before and after CXL treatment. No significant difference was seen at the 6- or 12-month follow-

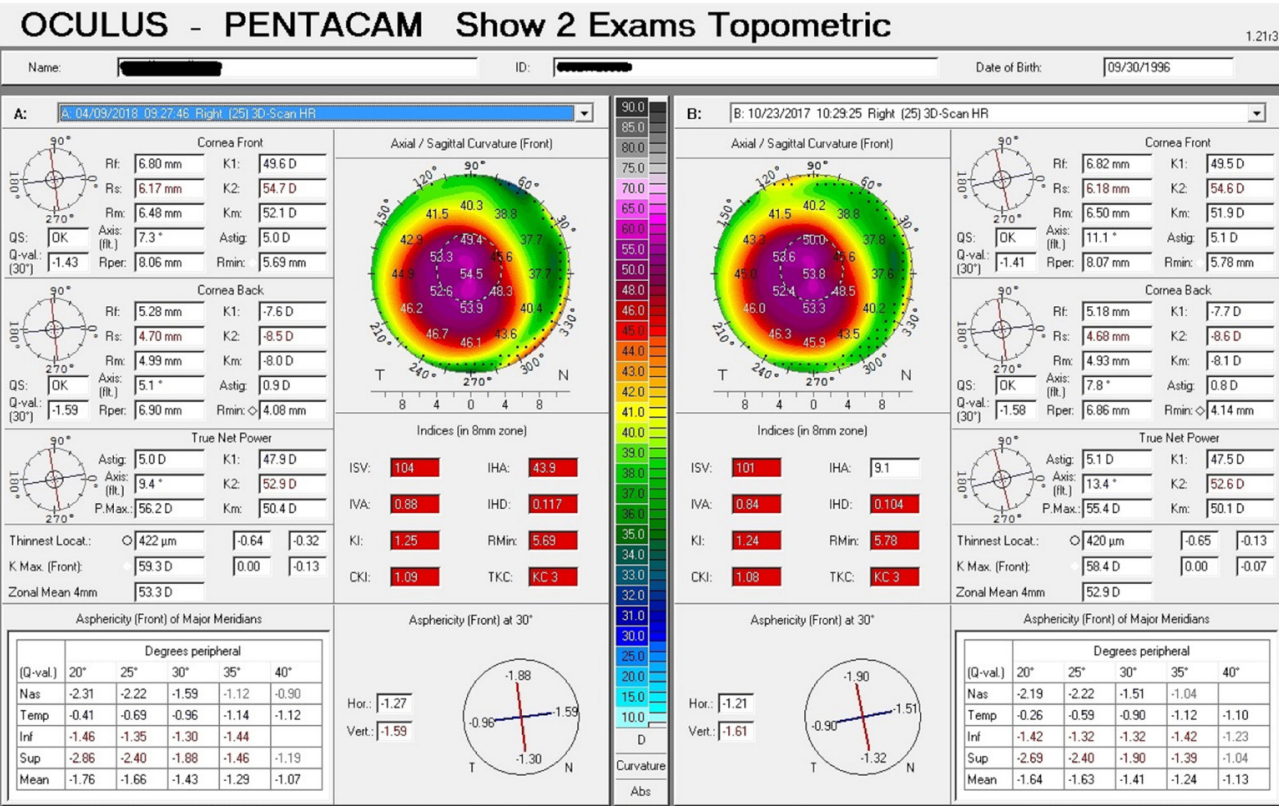

Figure 4. Corneal steepening of ID in the maximum keratometry observed 12 months after corneal cross-linking treatment. 
up examination in either parameter.

To the best of our knowledge, there is no study in the literature that has examined EKR values after CXL treatment; however, Holladay EKR readings have been analyzed in patients who underwent corneal refractive surgery, such as photorefractive keratectomy (PRK), LASIK, and LASEK. Hua et al. (23) examined a post-LASIK patient group and determined that there was a consistent reduction from the I-mm EKR (EKRI) measurement until EKR4, followed by an increase to EKR 7. Falavarjani et al. (24) determined a correlation between SimK and EKR4 in patients who were treated with PRK for myopia, and Savini et al. (25) found a difference between SimK and EKR in all measurements. However, in those studies, the data used were obtained from eyes that had undergone corneal refractive surgery, and only the repeatability of the postoperative values was evaluated, as no comparison of before and after treatment was made. Furthermore, in a study of Holladay EKR in keratoconus by Saglik et al. (I2), it was reported that the error margin was high in measurements of $I \mathrm{~mm}$ to $3 \mathrm{~mm}$ because of the change of corneal apex centralization.

In the current study, a curve was drawn showing a logarithmic reduction from EKRI to EKR7 (Fig. I). The SimK values declined in a similar way but the curve was more flattened in the $4 \mathrm{~mm}$ to $5 \mathrm{~mm}$ range (Fig. 2). Although there was a decrease in all of the measurement intervals of the Holladay EKR and SimK measurements at 6 and 12 months postoperatively compared with the preoperative values, it was not statistically significant. There was an evident improvement in the Holladay EKR values of the mild keratoconus group after treatment, suggesting that $\mathrm{CXL}$ treatment led to better corneal improvements in mild keratoconus (Fig. I). In the moderate and severe groups, the postoperative curve was parallel with the preoperative values.

The keratometric results of corneal collagen CXL treatment have varied in different studies. Although a reduction in $\mathrm{Kmax}$ and mean $\mathrm{K}$ values has been shown in several studies in the literature in measurements made with different topography devices in a 12 -month follow-up period $(16,17)$, in some studies with a small number of patients, increases have been determined in Kmax values, as described by Hersh et al., who reported $4.2 \%$, and by Asri et al., (22) who found a change of $9.8 \%$. In the current study, the rate was determined to be $2 \%$. In the 12-month follow-up of this study, an increase of >ID in the Kmax values was seen in I case and no correlation was seen with other parameters of progression. It was thought that these values would decline over the subsequent follow-up period.

Kmax and SimK values in the heat map demonstrated homogeneous distribution in the mild and severe groups, while the EKR values were not homogeneously distributed in any group (Fig. 3). In some cases, there was no correlation between Kmax values and other measurements. It is possible that the difference was due to the peripheral location of the corneal apex.

Since Wollensak et al. (8) first reported the results of CXL treatment, many new treatment modalities have been developed and the efficacy and reliability of these have been investigated with confocal microscopy $(26,27)$. It has been reported that CXL treatment caused keratocyte apoptosis, especially in the anterior corneal stroma, and the treatment has been seen to be effective at $350 \mu \mathrm{m}$ in the corneal stroma. Furthermore the posterior corneal surface curvature can be affected by the treatment (28). Since Holladay EKR measurements reflect both the anterior and posterior surface, it represents a further step forward.

A limitation of this study was the small number of patients in the study group. There is a need for further studies with a more extensive patient series using more topographic parameters.

In conclusion, the results of this study showed that the Holladay EKR measurements differed from the SimK measurements, and particularly in mild keratoconus. In addition to anterior corneal surface measurements, methods that can evaluate anterior and posterior surface curvatures can provide more accurate results in progression analysis.

\section{Disclosures}

Ethics Committee Approval: Approval for the study was granted by the Ethics Committee of the Faculty of Medicine of Harran University, Şanlıurfa, Turkey. All of the study procedures were performed in compliance with the Helsinki Declaration.

Peer-review: Externally peer-reviewed.

Conflict of Interest: The authors declare that we have no competing interests.

Authorship Contributions: Involved in design and conduct of the study (AS, MA); preparation and review of the study (AS, HC); data collection (AS, MA); and statistical analysis (AS, HC).

\section{References}

I. Rabinowitz YS. Keratoconus. Surv Ophthalmol 1998;42:297319. [CrossRef]

2. Martínez-Abad A, Piñero DP. New perspectives on the detection and progression of keratoconus. J Cataract Refract Surg 2017;43:12।3-27. [CrossRef]

3. Wollensak G, Spörl E, Seiler T. Treatment of keratoconus by collagen cross linking. [Article in German]. Ophthalmologe 2003;100:44-9. [CrossRef]

4. Wittig-Silva C, Chan E, Islam FM, Wu T, Whiting M, Snibson GR. A randomized, controlled trial of corneal collagen crosslinking in progressive keratoconus: three-year results. Ophthalmology 2014;121:8|2-2I. [CrossRef]

5. Randleman JB, Khandelwal SS, Hafezi F. Corneal cross-linking. 
Surv Ophthalmol 2015;60:509-23. [CrossRef]

6. Asgari S, Hashemi H. OPD scan III accuracy: Topographic and aberrometric indices afteraccelerated corneal cross-linking. J Curr Ophthalmol 2017;30:58-62. [CrossRef]

7. Raiskup F, Theuring A, Pillunat LE, Spoerl E. Corneal collagen crosslinking with riboflavin and ultraviolet-A light in progressive keratoconus: ten-year results. J Cataract Refract Surg 20I5;4I:4I-6. [CrossRef]

8. Wollensak G, Spoerl E, Seiler T. Riboflavin/ultraviolet-a-induced collagen crosslinking for the treatment of keratoconus. Am J Ophthalmol 2003; 135:620-7. [CrossRef]

9. Koller T, Pajic B, Vinciguerra P, Seiler T. Flattening of the cornea after collagen crosslinking for keratoconus. J Cataract Refract Surg 201 I;37: I488-92. [CrossRef]

10. Holladay JT, Hill WE, Steinmueller A. Corneal power measurements using scheimpflug imaging in eyes with prior corneal refractive surgery. J Refract Surg 2009;25:862-8. [CrossRef]

I I. Tang Q, Hoffer KJ, Olson MD, Miller KM. Accuracy of Scheimpflug Holladay equivalent keratometry readings after corneal refractive surgery. J Cataract Refract Surg 2009;35: I 198-203. [CrossRef]

12. Saglik A, Celik H. Comparison of Holladay equivalent keratometry readings and anteriorcorneal surface keratometry measurements in keratoconus. Int Ophthalmol 2019;39:150I-9.

13. Alió JL, Shabayek MH. Corneal higher order aberrations: a method to grade keratoconus. J Refract Surg 2006;22:539-45.

14. Ho JD, Tsai CY, Tsai RJ, Kuo LL, Tsai IL, Liou SW. Validity of the keratometric index: evaluation by the Pentacam rotating Scheimpflug camera. J Cataract Refract Surg 2008;34:137-45.

I5. Wang L, Mahmoud AM, Anderson BL, Koch DD, Roberts CJ. Total corneal power estimation: ray tracing method versus gaussian optics formula. Invest Ophthalmol Vis Sci 201 I;52:1716-22.

16. Hashemi H, Seyedian MA, Miraftab M, Fotouhi A, Asgari S. Corneal collagen cross-linking with riboflavin and ultraviolet a irradiation for keratoconus: long-term results. Ophthalmology 2013;| 20:1515-20. [CrossRef]

17. Vinciguerra P, Albè E, Trazza S, Rosetta P, Vinciguerra R, Seiler $\mathrm{T}$, et al. Refractive, topographic, tomographic, and aberrometric analysis of keratoconic eyes undergoing corneal cross-linking. Ophthalmology 2009; I 16:369-78. [CrossRef]
18. Caporossi A, Mazzotta C, Baiocchi S, Caporossi T. Long-term results of riboflavin ultraviolet a corneal collagen cross-linking for keratoconus in Italy: the Siena eye cross study. Am J Ophthalmol 2010; 149:585-93. [CrossRef]

19. Hersh PS, Greenstein SA, Fry KL. Corneal collagen crosslinking for keratoconus and corneal ectasia: One-year results. J Cataract Refract Surg 201 I;37:149-60. [CrossRef]

20. Koller T, Iseli HP, Hafezi F, Vinciguerra P, Seiler T. Scheimpflug imaging of corneas after collagen cross-linking. Cornea 2009;28:510-5. [CrossRef]

2I. Zotta PG, Diakonis VF, Kymionis GD, Grentzelos M, Moschou $\mathrm{KA}$. Long-term outcomes of corneal cross-linking for keratoconus in pediatric patients. J AAPOS 2017;21:397-40I. [CrossRef]

22. Asri D, Touboul D, Fournié P, Malet F, Garra C, Gallois A, et al. Corneal collagen crosslinking in progressive keratoconus: multicenter results from the French National Reference Center for Keratoconus. J Cataract Refract Surg 20I I;37:2137-43. [CrossRef]

23. Hua Y, Zhang X, Utheim TP, Huang J, Pan C, Tan W, et al. Evaluation of Equivalent Keratometry Readings Obtained by Pentacam HR (High Resolution). PLoS One 2016; I I:e0I50I2I. [CrossRef]

24. Falavarjani KG, Hashemi M, Joshaghani M, Azadi P, Ghaempanah MJ, Aghai GH. Determining corneal power using Pentacam after myopic photorefractive keratectomy. Clin Exp Ophthalmol 2010;38:34I-5. [CrossRef]

25. Savini G, Barboni P, Profazio V, Zanini M, Hoffer KJ. Corneal power measurements with the Pentacam Scheimpflug camera after myopic excimer laser surgery. J Cataract Refract Surg 2008;34:809-13. [CrossRef]

26. Mazzotta C, Hafezi F, Kymionis G, Caragiuli S, Jacob S, Traversi C, et al. In Vivo Confocal Microscopy after Corneal Collagen Crosslinking. Ocul Surf 2015;13:298-314. [CrossRef]

27. Bouheraoua N, Jouve L, El Sanharawi M, Sandali O, Temstet $\mathrm{C}$, Loriaut $\mathrm{P}$, et al. Optical coherence tomography and confocal microscopy following three different protocols of corneal collagen-crosslinking in keratoconus. Invest Ophthalmol Vis Sci 2014;55:760I-9. [CrossRef]

28. Steinberg J, Ahmadiyar M, Rost A, Frings A, Filev F, Katz T, et al. Anterior and posterior corneal changes after crosslinking for keratoconus. Optom Vis Sci 2014;91:178-86. [CrossRef] 